Rosana Teresa Onocko Campos'

Juarez Pereira Furtado"

Eduardo Passos ${ }^{\text {III }}$

Ana Luiza Ferrer ${ }^{\text {IV }}$

Lilian Miranda ${ }^{\text {IV }}$

Carlos Alberto Pegolo da

Gama $^{\text {IV }}$

\section{Avaliação da rede de centros de atenção psicossocial: entre a saúde coletiva e a saúde mental}

\author{
Evaluation of the network of \\ psychosocial care centers: between \\ collective and mental health
}

Departamento de Medicina Preventiva e Social. Faculdade de Ciências Médicas. Universidade Estadual de Campinas (UNICAMP). Campinas, SP, Brasil

" Universidade Federal de São Paulo. São Paulo, SP, Brasil

III Universidade Federal Fluminense. Rio de Janeiro, RJ, Brasil

Programa de Pós-Graduação em Saúde Coletiva. UNICAMP. Campinas, SP, Brasil

Correspondência | Correspondence:

Rosana Teresa Onocko-Campos

Departamento de Medicina Preventiva e Social

Universidade Estadual de Campinas

Caixa Postal 6111

Cidade Universitária Zeferino Vaz - Barão

Geraldo

13081-970 Campinas, SP, Brasil

E-mail: rosanaoc@mpc.com.br

\section{RESUMO}

OBJETIVO: Avaliar os modelos assistenciais, de gestão e de formação de trabalhadores de uma rede centros de atenção psicossocial (CAPS).

MÉTODOS: Pesquisa avaliativa qualitativa, sustentada pela hermenêutica gadameriana, realizada na cidade de Campinas (SP), em 2006-2007. Os dados foram coletados por meio de 20 grupos focais, em CAPS III, realizados com diferentes grupos de interesse (trabalhadores, gestores municipais, usuários, familiares e gestores locais). Após a transcrição do material gravado de cada grupo, foram construídas narrativas, seguindo o referencial teórico de Ricoeur. $\mathrm{Na}$ segunda etapa de grupos focais essas narrativas foram apresentadas aos participantes para contestá-las, corrigi-las e validá-las. Os resultados preliminares foram discutidos em oficinas para elaboração de um guia de boas práticas em CAPS III.

RESULTADOS: Foram identificados pontos fortes e fragilidades no que concerne à atenção à crise, articulação com a rede básica, formulação de projetos terapêuticos, gestão e organização em equipes de referência, formação educacional e sofrimento psíquico.

CONCLUSÕES: A rede de centros de atenção psicossocial em Campinas destaca-se pela sua originalidade na implantação de seis CAPS III , e pela sua eficácia na continência com usuários e familiares no momento da crise e na reabilitação. A organização por técnico e/ou equipe de referência prevalece, assim como a construção de projetos terapêuticos. A redução das equipes noturnas desponta como principal problema e fonte de estresse para os trabalhadores. A formação dos profissionais se mostrou insuficiente para os desafios enfrentados por esses serviços.

DESCRITORES: Serviços de Saúde Mental. Pessoal de Saúde. Gestão em Saúde. Reforma dos Serviços de Saúde. Pesquisa Qualitativa. Pesquisa Avaliativa. 


\section{ABSTRACT}

OBJECTIVE: To analyze the assistance, management and workers' education models of a network of psychosocial healthcare services (CAPS).

METHODS: This is a qualitative evaluation research, supported by the Gadamerian hermeneutics, carried out in the city of Campinas, Southeastern Brazil, in 2006-2007. Data were collected through 20 focus groups in centers known as CAPS III, with different groups of stakeholders (workers, municipal managers, users, family members and local managers). After the transcription of each group's recorded material, narratives were constructed following Ricoeur's theoretical framework. At the second stage of the focus groups, these narratives were presented to the participants, who could contest, correct and validate them. The preliminary results were discussed in workshops, with the aim of developing a good practice guide in CAPS III.

RESULTS: The study identified strong points and weaknesses concerning the care provided during the crisis, articulation with the primary care network, formulation of therapeutic projects, management and organization in reference teams, educational background and psychological distress.

CONCLUSIONS: The network of psychosocial care centers in Campinas stands out due to its originality in the implementation of six CAPS III and to its efficacy in providing comprehensive assistance to users and family members in the moment of crisis and in rehabilitation. The organization in reference technician and/or team prevails, as well as the development of therapeutic projects. Night teams reduction is the most important problem and the main source of workers' stress. The professionals' education proved to be insufficient to deal with the challenges faced by these services.

\section{DESCRIPTORS: Mental Health Services. Health Personnel. Health Management. Health Care Reform. Qualitative Research. Evaluation Research.}

\section{INTRODUÇÃO}

Os centros de atenção psicossocial (CAPS) são considerados serviços estratégicos para a organização da rede de atenção à saúde mental em um determinado território e para a consolidação da Reforma Psiquiátrica brasileira. Sua implantação e qualificação vêm sendo incentivadas pelo Ministério da Saúde, de tal modo que o número de centros mais do que dobrou nos últimos cinco anos anos. Além disso, algumas portarias foram criadas para incrementar as políticas de financiamento e qualificação assistencial. ${ }^{\text {a }}$

Na condição de serviços públicos de saúde mental no campo da saúde coletiva, ${ }^{1,3}$ devem garantir acesso, integralidade e resolutividade na assistência prestada, agregando os diferentes níveis de atenção (primário, secundário e terciário) em uma unidade, gerando reflexões concernentes ao modelo assistencial e à clínica ${ }^{10}$ A complexidade na organização da assistência e os impactos subjetivos nos profissionais próprios do trabalho com a psicose $\mathrm{e}^{7,8}$ conferem ao CAPS desafios e necessidade de constante acompanhamento e análise. Os aspectos institucionais da Reforma Psiquiátrica, atualizados nos CAPS, apresentam relações entre intersubjetividade, gestão, formação profissional e clínica. ${ }^{10}$ Assim, é necessária uma abordagem avaliativa que contemple a pluralidade e interdisciplinaridade de tais relações.

O presente estudo teve por objetivo analisar os modelos assistenciais, de gestão e de formação dos trabalhadores de uma rede de centros de atenção psicossocial.

\section{MÉTODOS}

O estudo constitui uma pesquisa avaliativa, conduzida entre 2006-2007, na qual associamos temáticas e categorias advindas da saúde coletiva, tais como gestão e subjetividade, ${ }^{11,12}$ com a avaliação qualitativa, 
participativa e construtivista, 2,4,6 tendo em vista a institucionalização das políticas públicas de saúde mental para os novos serviços ${ }^{3}$ e às vicissitudes identificadas pelos trabalhadores e usuários em seus cotidianos de trabalho ou na freqüência aos serviços.

A abordagem foi qualitativa com referencial teórico apoiado na hermenêutica gadameriana. ${ }^{5} \mathrm{O}$ trabalho de campo foi desenvolvido na cidade de Campinas (SP) em função da complexidade e extensão do seu sistema de saúde mental e do pioneirismo na implantação de seis CAPS III (com acolhimento diuturno).

Os dados foram coletados por meio de 20 grupos focais, ${ }^{9}$ realizados com os grupos de interesse (stakeholders): ${ }^{14}$ 12 grupos com trabalhadores de cada um dos seis CAPS, dois grupos com o colegiado municipal de saúde mental, dois grupos com os gerentes dos CAPS envolvidos, dois grupos com familiares de usuários dos seis CAPS e dois grupos com usuários. Esses grupos participaram de duas etapas. Na primeira, havia um roteiro com questões disparadoras da discussão, abrangendo as temáticas de interesse da pesquisa, sendo os roteiros diferentes em alguns aspectos segundo o grupo de interesse. Após a transcrição do material gravado desses grupos, foram construídas narrativas ${ }^{13}$ de cada um deles, seguindo o referencial teórico de Ricoeur. ${ }^{15,16}$ A construção narrativa consistiu no encadeamento dos núcleos argumentais presentes no material transcrito, mas editado à maneira de uma história que se deixa seguir, na leitura. Na segunda etapa essas narrativas foram apresentadas aos mesmos participantes dos grupos focais, que puderam contestá-las, corrigi-las e validá-las. Houve perda insignificante de sujeitos entre uma etapa e outra (três pessoas de um total de 120).

Após transcrição do material da segunda etapa de grupos focais, foram construídas novas narrativas, que, juntamente com aquelas dos primeiros grupos, foram analisadas e constituíram-se material-base para as oficinas de construção de parâmetros e dispositivos avaliativos, que aconteceram posteriormente.

As oficinas objetivaram o processamento dos resultados da pesquisa. Para tanto, a participação foi ampliada, incluindo, além dos grupos de interesse descritos para a realização dos grupos focais, trabalhadores de outros serviços da rede de saúde, como Serviço de Atendimento Móvel de Urgência (SAMU), unidades básicas de saúde, centros de convivência, supervisores de CAPS, abrangendo cerca de 300 participantes ao total. Procuramos assim valer-nos de uma técnica de criação de consenso que diversificasse e ampliasse a participação e não da já consagrada técnica de Delphi. ${ }^{17} \mathrm{O}$ objetivo de nossa inovação foi radicalizar a pactuação dialógica entre os múltiplos grupos de interesse envolvidos.

Foram realizadas dez oficinas em duas sessões, divididas pelas seguintes temáticas: 1) Concepção de
CAPS, 2) Projeto terapêutico individual e práticas grupais, 3) Atenção à crise, 4) Formação profissional e sofrimento dos trabalhadores e 5) Gestão. O tema das relações dos CAPS com a rede de serviços de saúde em geral permaneceu transversal a todas as oficinas. Na primeira sessão, apresentamos aos participantes uma lista de problemas, controvérsias e soluções relativos a cada tema que identificamos nas narrativas dos grupos focais; os participantes elegeram quais necessitariam de monitoramento avaliativo. Na segunda sessão, as mesmas oficinas procederam à elaboração de parâmetros e dispositivos avaliativos para o monitoramento.

A pesquisa foi aprovada pelo Comitê de Ética em Pesquisa da Universidade Estadual de Campinas (Processo No 396/2004). Todos os sujeitos assinaram o termo de consentimento livre e esclarecido.

\section{ANALISE E DISCUSSÃO DOS RESULTADOS}

\section{Formas de atenção ao usuário em momentos de crise}

Os participantes avaliaram como positiva a permanência do usuário com a mesma equipe nos momentos de crise, fato permitido pela existência de leitos nos CAPS III, sendo um importante diferencial em relação ao modelo hospitalar. Usuários e familiares consideraram continente a rede de saúde mental (em especial CAPS e SAMU). Foram relatadas como dificuldades: os entraves na formação de parcerias com a rede como um todo, o recebimento e acompanhamento de pacientes ainda desconhecidos pela equipe, sobretudo à noite e nos fins de semana, pois nesses períodos críticos costumam trabalhar no CAPS apenas profissionais de enfermagem, em esquema de plantão. Ainda, relatou-se como necessário objetivar os critérios de indicação de leito-noite, que tem sido utilizado no limite ou acima de sua capacidade durante o período em estudo.

Foram indicadas duas concepções antagônicas de crise: uma ligada à emergência de sintomas a serem contidos e outra que compreende o momento como de extrema fragilidade e oportunidade de reconstruções. Não havia acompanhamento sistemático dos usuários internados em leitos psiquiátricos fora do CAPS. Ainda, usuários e familiares queixaram-se do desaparecimento de pertences pessoais durante utilização do leito-noite.

Como parâmetros avaliativos, foi indicada a verificação periódica da taxa de ocupação e permanência nos leitos, bem como percentagens referentes a: atendimentos a pacientes em crise sem ocupação de leitos, usuários encaminhados para uso de leitos em outros serviços, usuários com diagnóstico de co-morbidade que demandam acompanhamento intensivo.

Como dispositivos avaliativos, os participantes suge- 
riram a criação de um grupo de apoio composto por profissionais dos vários CAPS a ser acionado para avaliação de risco quando uma equipe considerar a possibilidade de encaminhar algum usuário para internação em hospital.

Os participantes das oficinas ainda propuseram que os serviços avaliem a dosagem e eventuais associações entre medicações, revisão da prescrição em momentos de crise, avaliação dos pacientes em uso de leitonoite, intensificação do acompanhamento oferecido às famílias, estabelecimento de comunicação entre os profissionais sobre os motivos do uso do leito e da alta, e compartilhamento das decisões tomadas durante a crise com a família. Foi também proposto explicitar os critérios de encaminhamento para internação em outros serviços, assim como garantir o acompanhamento de eventuais internações, além de recomendarem a disponibilidade do serviço para a atenção domiciliar aos pacientes em crise.

\section{Projeto Terapêutico Individual (PTI) e Profissional/Equipe de Referência}

Na avaliação desse tema, o PTI foi considerado como consolidado nos serviços, sendo avaliado periodicamente e com o cuidado de não torná-lo protocolar. O trabalho em equipes de referência foi preferido por permitir e melhorar a discussão dos casos. Considerando-se a centralização das práticas de acompanhamento no profissional de referência, importantes relações de confiança puderam ser constituídas, entretanto, poderia haver dificuldade de compartilhamento de responsabilidades pelo caso. Relatou-se número excessivo de usuários por profissional de referência.

Quanto aos parâmetros avaliativos, indicou-se verificação periódica da percentagem de usuários que: utilizam outros recursos da rede, tiveram seu PTI discutido no último ano, foram atendidos por outros profissionais, além do seu técnico de referência.

No que se refere aos dispositivos avaliativos, foram sugeridas as criações de oficinas para os trabalhadores com objetivo de apresentar e discutir o trabalho que fazem e o referencial teórico que adotam e de oficinas voltadas para usuários e familiares, com o objetivo de esclarecê-los acerca dos principais medicamentos utilizados e das formas de estruturação do serviço.

\section{Práticas de grupo}

Os participantes avaliaram que tais práticas acontecem em todos os CAPS, sob diversas modalidades, porém não são discutidas ou supervisionadas entre os profissionais. Já os usuários referiram-se aos grupos como importante espaço de aprendizagem e trocas, enquanto os familiares descreveram-nos como oportunidades de suporte, mas gostariam que acontecessem em horários mais convenientes e com a presença do técnico de referência de seu familiar.

Em relação aos parâmetros avaliativos, foram relatados: planejamento de recursos e competências necessárias para desenvolver as práticas grupais; a existência de grupos para familiares e critérios de participação, bem como à inclusão de usuários na construção e avaliação dos grupos oferecidos, mapeamento dos grupos existentes dentro e fora do território do CAPS, e o estabelecimento de parcerias com outros serviços da rede para a realização de grupos.

Não foram mencionados dispositivos avaliativos.

\section{Gestão}

Fizeram parte da avaliação os relatos referentes ao reconhecimento da responsabilidade do gestor com a clínica desenvolvida, o reconhecimento das passagens de plantão como dispositivo privilegiado de contato com o cotidiano do serviço e a crítica ao modo de funcionamento dos noturnos estruturarem-se de maneira semelhante às rotinas dos hospitais psiquiátricos. Os participantes mencionaram a inexistência de plano de cargos e salários, isonomia e avaliação dos trabalhadores. As supervisões clínicas e institucionais, embora muito valorizadas pelos trabalhadores prescindiam de instâncias de acompanhamento e avaliação. Relataram-se dificuldades de gerenciamento de médicos por profissionais não médicos e dificuldades decorrentes do oferecimento de assistência $24 \mathrm{~h}$ de enfermagem, mas não de equipe multidisciplinar.

Foram citados como parâmetros avaliativos a participação regular dos gestores dos CAPS nos fóruns intersetoriais, nas intervenções do CAPS no território, nas atividades que incluem familiares e a existência de conselho gestor e outros arranjos participativos da gestão, como as assembléias.

Para a avaliação da gestão dos CAPS, propôs-se ainda que os serviços questionem-se sobre a participação dos gestores nas decisões clínicas e na construção dos PTI, sobre a existência de supervisão clínico-institucional, de formação continuada, de atenção interdisciplinar na atenção à crise durante a noite e nos fins de semana e de participação da equipe noturna na discussão dos PTI.

\section{Concepção de CAPS e articulação com a rede básica}

Os participantes avaliaram que alguns CAPS definiam-se como alternativos ao manicômio e outros como substitutivos, sendo diferentes entre si quanto ao número de internações psiquiátricas que indicam. Houve diferenças também quanto à construção de parceria com a Rede (potencializada nos CAPS sob gestão direta da secretaria municipal de saúde) e quanto à gestão do coti- 
diano (facilitada nos CAPS sob administração de uma organização não-governamental, em co-gestão com a prefeitura). Os coordenadores de unidades básicas se mostraram como tendo idéia vaga e distorcida sobre o trabalho do CAPS, independentemente do número de pacientes que sua unidade encaminhou para a Saúde Mental. Os agentes de saúde estudados desconheciam a função dos CAPS e afirmaram praticar ações de saúde mental baseadas em senso comum.

Foi indicada como parâmetro avaliativo a identificação do número de serviços que compartilham projetos com o CAPS e como dispositivos avaliativos: criação de um fórum regular dos CAPS, onde esses serviços pudessem compartilhar problemas e soluções, constituição de conselhos locais nos CAPS e registro dos encontros e da prestação de contas realizados nos serviços.

Sobre a relação do CAPS com a rede, as oficinas ainda propuseram que os serviços conhecessem as concepções que as UBS possuem do trabalho dos CAPS, fizessem o mapeamento do percurso do usuário pela rede até chegar ao CAPS, adequassem os CAPS à Portaria 336 (que regulamenta os CAPS) e melhorassem a qualidade da alimentação oferecida aos usuários.

\section{Formação educacional e sofrimento psíquico dos trabalhadores}

Os participantes avaliaram como fontes de sofrimento: contato com a loucura e com as carências sociais dos usuários, grande demanda, falta de recursos, dificuldades de diálogo com a rede básica, falta de discriminação entre os espaços abertos aos usuários e aqueles privativos aos trabalhadores e, dificuldades de relacionamento na equipe em relação à hierarquia institucional, principalmente para os profissionais de enfermagem de nível médio.

A organização dos plantões noturnos e de fins de semana foi relatada como maior fonte angústia, pois os profissionais permanecem em contato direto com os pacientes em crise por períodos prolongados, tomando sozinhos decisões importantes sobre os casos. Várias doenças (hipertensão, depressão, entre outras) foram associadas a somatizações do sofrimento pelo/no trabalho. As passagens de plantões, reuniões de equipe e supervisões foram referidas com formas de auxiliar a lidar com o sofrimento.

Indicou-se como parâmetro avaliativo a percentagem de trabalhadores envolvidos com algum tipo de formação profissional, bem como daqueles que recebem subsídios da instituição para tal.

Dentre os aspectos propostos para serem levados em conta na avaliação do serviço, houve participantes que salientaram a importância de se refletir sobre a existência e a duração das reuniões de equipe, a participação de todos nas decisões coletivas, o diálogo entre as diversas abordagens clínicas, as estratégias utilizadas para a recepção e o acompanhamento de usuários, a existência, regularidade e garantia de financiamento das supervisões, a adequação da linguagem utilizada na supervisão aos diversos integrantes da equipe, bem como sua capacidade de provocar um auto-questionamento na equipe em questão e de discutir os PTI.

As oficinas propuseram também estimular a realização de passagem de plantão entre as equipes do dia e da noite, questionar o paradigma que sustenta o trabalho da noite, averiguar a coerência entre as equipes diurna e noturna acerca dos critérios de indicação do leitonoite e a possibilidade de a equipe noturna participar das reuniões de equipe e ter suas questões consideradas. Enfatizou-se a necessidade de capacitações de qualidade com critérios transparentes de ingresso quando financiadas pelo poder público, de flexibilidade da gestão para a participação dos trabalhadores nos processos de formação permanente e de apoio para que os profissionais também possam tornar-se formadores, transmitindo sua experiência.

\section{CONSIDERAÇÕES FINAIS E RECOMENDAÇÕES}

Em Campinas, a expansão da rede de CAPS ocorreu em virtude do esforço e da coragem de gestores e trabalhadores em suas mais variadas formações produzindo: reabilitação, acompanhamento em meio comunitário, favorecimento da autonomia e efetiva superação do modelo centrado no hospital psiquiátrico. Esse efeito das políticas públicas de saúde mental não pode ser menosprezado na discussão sobre recursos.

As fragilidades observadas na rede de saúde mental de Campinas apontam a necessidade de oferecer elementos normativos que viabilizem a extensão da assistência noturna e de fim de semana à equipe multiprofissional como um todo. A sua falta coloca os CAPS III em risco de repetirem a fragmentação dia/noite, velha e desagradável conhecida do hospital psiquiátrico. Em Campinas, o fato de os médicos estarem de plantão à distância (SAMU) é, por um lado, fonte de retaguarda para a rede como um todo e, por outro, fonte de intenso estresse quando se trata de pacientes não conhecidos pelas equipes ou com potenciais problemas clínicos não psiquiátricos associados.

Algumas recomendações podem ser formuladas a partir dos achados de nossa investigação e as oferecemos como subsidio para a necessária e permanente reformulação das políticas públicas da área:

a Ministério da Saúde. Legislação em saúde mental. 2. ed. Brasília; 2002. 
- urge expandir tanto os cursos de especialização como redefinir os planos curriculares de várias profissões da saúde;

- é importante definir planos de cargos e salários, processos seletivos e normas de estabilidade que permitam qualificar e retomar a necessária isonomia no trabalho nos CAPS;

- a formação continuada dos profissionais dos CAPS deveria ser estimulada. Em relação a isso, as supervisões clínico-institucionais são escassas e existe o risco de transformar-se em espaço de "saber-poder" que opera sobre as equipes, com total descolamento da gestão local;

- o estímulo e o reforço à gestão democrática e à formação de gestores também devem ser considerados: ao gestor local cabe, afinal, a gestão da clínica do CAPS, de suas relações com a rede de saúde como um todo e da própria equipe multiprofissional;

- os usuários e familiares deveriam contar com mais informações viabilizadas pelos serviços públicos de saúde em relação às doenças, aos tratamentos e às medicações, fortalecendo sua participação e autonomia;

- é importante debater a temática da porta de entrada dos CAPS e de sua inserção no território. Familiares e usuários destacam a importância dos serviços serem plásticos e adaptados às suas possibilidades, possibilitando atender urgências: horários das reuniões, atenção à crise em domicílio, por exemplo;

- os CAPS fazem parte de uma política eficaz de saúde mental voltada para os pacientes psicóticos e neuróticos muito graves que tenham ou não atravessado pela experiência de ser asilado. Esta política deve ser ampliada e sustentada garantindo sua viabilidade em termos de recursos, capacitação e contratações;

- é necessário o desenho de uma política pública voltada para outros problemas prevalentes de saúde mental, como caracterizam as crises subjetivas de outra ordem: violência, somatizações, depressão, drogadicção dentre outros, de maneira a definir os papéis e os perfis das várias instâncias envolvidas na assistência à saúde mental.

Em nossa pesquisa, os CAPS mostraram-se um campo rico em práticas e sistematizações que indicam avanços consolidados para a organização do serviço. A inclusão de trabalhadores, familiares e usuários tanto nos grupos focais como nas oficinas potencializou os dispositivos da pesquisa participativa e outorgou ao material nelas produzido uma ênfase na diversidade, ao respeito pela diferença, ao reconhecimento da expertise de vida e não somente da academia ou da gestão de serviços.

A inovação metodológica do estudo está na utilização da abordagem narrativa para o tratamento do material produzido pelos grupos focais, assim como no posterior manuseio participativo destes textos: com a extração dos núcleos argumentais e sua transformação, nas oficinas, em indicadores e analisadores. Essas inovações foram provocadas pelo interesse dos pesquisadores ${ }^{5}$ em produzir intervenções dialógicas e também pelo próprio objeto de pesquisa, pois ainda há pouca literatura a respeito das "caixas-pretas" dos processos de trabalho nos CAPS.

A exploração das narrativas propiciou o aparecimento de histórias "não-ainda-narradas”. ${ }^{15}$ Com esta escolha, pretendíamos não somente contar uma história única e cronologicamente arranjada, mas, sobretudo, compreender os processos de trabalho e as trocas que acontecem no cotidiano dos CAPS.

Percebemos que, apesar das divergências discursivas, os trabalhadores puderam identificar muitos problemas comuns e necessidade de estratégias conjuntas para seu enfrentamento. Há atualmente um fórum de trabalhadores de CAPS constituindo-se em Campinas, o que será uma inovação para o processo de trabalho em saúde mental, para a qual nossa pesquisa contribuiu. Diz Gadamer ${ }^{5}$ que, quando se logra compreender, compreende-se sempre de maneira diferente, por isso produtiva.

Classicamente se afirma que a pesquisa qualitativa não produz explicações, senão interpretações que ampliam nossa compreensão do objeto em estudo. Suas possíveis generalizações não se colocam ao nível da empiria, mas ao teórico-conceitual. Nesse sentido, esperamos que os consensos sobre o que sejam analisadores, indicadores e parâmetros para uma rede de CAPS III possam ser de utilidade para outros pesquisadores e para os próprios gestores do SUS em suas esferas local e federal.

Nosso estudo de uma rede municipal de CAPS III permanece inédito, pela instalação recente desses serviços no Brasil e por não termos serviços semelhantes na bibliografia internacional, nem ainda outros estudos publicados no contexto brasileiro. A proposta que os CAPS III assumem de tratar a crise longe do hospital psiquiátrico é interesse de outros sistemas nacionais de saúde que estejam preocupados com a melhora da assistência à saúde mental.

Evidentemente, a compreensão que pudemos obter apresenta marcas características da rede de saúde mental de Campinas, de sua formação histórica e social. Contudo, nossa abordagem avaliativa, inspirada nas avaliações de quarta geração, ${ }^{6}$ permitiu-nos apreender as práticas dos CAPS na sua complexidade, compostas 
pelas várias opiniões e versões nas quais o que se coloca não é qual é a verdadeira, senão que, sendo todas verdadeiras elas exprimem um posicionamento singular no campo histórico-social. Assim, mais do que de possíveis

\section{REFERÊNCIAS}

1. Delgado PG, Gomes MPC, Coutinho ESF. Novos rumos nas políticas de saúde mental no Brasil. Cad Saude Publica. 2001;17(3):452-3. DOI: 10.1590/S0102311X2001000300001

2. Furtado JP. Um método construtivista para a avaliação em saúde. Cienc Saude Coletiva. 2001;6(1):165-82. DOI: 10.1590/S1413-81232001000100014

3. Furtado JP, Onocko Campos R. A transposição das políticas de saúde mental no Brasil para a prática nos novos serviços. Rev Latinoam Psicopatol Fundam. 2005;8(1):109-22.

4. Furtado JP. Avaliação para o conhecimento e a transformação. In: Bosi ML, Mercado F. Avaliação qualitativa em saúde. Rio de Janeiro: Vozes; 2006. p.191-206.

5. Gadamer HG. Verdade e método: traços fundamentais de uma hermenêutica filosófica. Petrópolis: Vozes; 1997.

6. Guba EG, Lincoln YS. Fourth generation evaluation. Newbury Park: Sage; 1989.

7. Kaës R. Realidade psíquica e sofrimento nas instituições. In: Kaës R, Bleger J, Enriquez E, Fornani F, Fustier P. A instituição e as instituições. São Paulo: Casa do Psicólogo; 1991.

8. Marazina I. Trabalhador da saúde mental: encruzilhada da loucura. SaudeLoucura. 1991;1(1):69-74. vieses caberia falar de limites de nossa investigação, limites dados pela metodologia que priorizou a compreensão à descrição objetiva, e a produção de consensos participativos à produção de verdades neutras.
9. Morgan DL. Focus groups as qualitative research. Thousand Oaks: Sage; 1997.

10. Onocko Campos R. Clínica: a palavra negada: sobre as práticas clínicas nos serviços substitutivos de saúde mental. Saude Debate. 2001;25(58):98-111.

11. Onocko Campos R. La gestion: espace d'interevention, d'analyse et de especificités techniques. Rev Prat Cah Med Utop. 2003;2(1):13954.

12. Onocko Campos R. O planejamento no labirinto: uma viagem hermenêutica. São Paulo: Hucitec; 2003.

13. Onocko Campos R. O encontro trabalhador-usuário na atenção à saúde: uma contribuição da narrativa psicanalítica ao tema do sujeito na saúde coletiva. Cien Saude Coletiva. 2005;10(3):573-83. DOI: 10.1590/S1413-81232005000300015

14. Patton MQ. Utilization-focused evaluation. Beverly Hills: Sage; 1997.

15. Ricoeur P. Interpretação e ideologias. Rio de Janeiro: Francisco Alves; 1990.

16. Ricoeur P. Tempo e narrativa. Campinas: Papirus; 1994. v.1.

17. Wright JTC, Giovinazzo RA. Delphi: uma ferramenta de apoio ao planejamento prospectivo. Cad Pesq Admin. 2000;1(12):55-65.

Pesquisa financiada pelo Ministério da Saúde e Conselho Nacional de Desenvolvimento Científico e Tecnológico (CNPq Proc. no 554426/2005-6).

Apresentado no II Seminário Internacional de Pesquisa de Serviços de Saúde Mental, realizado em Campinas, SP, em 2007. Artigo submetido ao processo de julgamento por pares adotado para qualquer outro manuscrito submetido a este periódico, com anonimato garantido entre autores e revisores. Editores e revisores declaram não haver conflito de interesses que pudesse afetar o processo de julgamento do artigo.

Os autores declaram não haver conflito de interesses 